\title{
Correction to: Environmental Noise Exposure and Neurodevelopmental and Mental Health Problems in Children: a Systematic Review
}

\author{
Fariba Zare Sakhvidi ${ }^{1} \cdot$ Mohammad Javad Zare Sakhvidi $^{2} \cdot$ Amir Houshang Mehrparvar $^{3} \cdot$ Angel M. Dzhambov $^{4}$
}

Published online: 27 July 2018

(C) Springer Nature Switzerland AG 2018

\section{Correction to: Current Environmental Health Reports https://doi.org/10.1007/s40572-018-0208-x}

The first author's name should be "Fariba Zare Sakhvidi".

The original article has been corrected.

The online version of the original article can be found at https://doi.org/ 10.1007/s40572-018-0208-x

Angel M. Dzhambov

angelleloti@gmail.com

1 Department of Biostatistics, School of Public Health, Shahid Sadoughi University of Medical Sciences, Yazd, Iran

2 Occupational Health Research Center, School of Public Health, Shahid Sadoughi University of Medical Sciences, Yazd, Iran

3 Industrial Diseases Research Center, Faculty of Medicine, Shahid Sadoughi University of Medical Sciences, Yazd, Iran

4 Department of Hygiene and Ecomedicine, Faculty of Public Health, Medical University of Plovdiv, 15-A Vassil Aprilov Blvd., 4002 Plovdiv, Bulgaria 